\title{
BMJ Open Quality Reducing two-unit red cell transfusions on the oncology ward: a choosing wisely initiative
}

\author{
Alan Gob, ${ }^{1}$ Anurag Bhalla, ${ }^{1,2}$ Laura Aseltine, ${ }^{3}$ Ian Chin-Yee ${ }^{2,3}$
}

To cite: Gob A, Bhalla A, Aseltine $\mathrm{L}$, et al. Reducing two-unit red cell transfusions on the oncology ward: a choosing wisely initiative. BMJ Open Quality 2019;8:e000521. doi:10.1136/ bmjoq-2018-000521

Received 30 August 2018 Revised 2 February 2019 Accepted 19 February 2019

Check for updates

(c) Author(s) (or their employer(s)) 2019. Re-use permitted under CC BY-NC. No commercial re-use. See rights and permissions. Published by BMJ.

${ }^{1}$ Medicine, London Health Sciences Centre, London, Ontario, Canada

${ }^{2}$ Medicine, University of Western Ontario, London, Ontario,

Canada

${ }^{3}$ Pathology and Laboratory

Medicine, London Health

Sciences Centre, London,

Ontario, Canada

Correspondence to

Dr Alan Gob;

Alan.Gob@lhsc.on.ca

\section{ABSTRACT}

Background/context Despite Choosing Wisely recommendations for single unit red blood cell transfusion orders, $\sim 50 \%$ of orders on the oncology ward at London Health Sciences Centre (LHSC) were for two units. The oncology ward at LHSC is a 60 bed tertiary care unit. In mid 2016, LHSC was 18 months into its implementation of computerised provider order entry (CPOE).

Aim/objectives By December 2017, increase the proportion of one-unit red cell transfusion orders on the oncology ward from $50 \%$ to $80 \%$

Measures Outcome: \% one-unit red cell transfusion orders (aggregated monthly).

Improvement/innovation/change ideas Our initial theory was that unawareness of the guidelines (established in 2014) and subscription to the obsolete doctrine of two-unit transfusions were the primary behavioural drivers. Initial change ideas included an educational/awareness blitz including rounds presentations, memos and posters. Failure led us to revisit our hypothesis and carry out a real-time audit, where our team was notified on each two-unit transfusion. This revealed the true root cause: the overwhelming majority of two-unit transfusions could be traced back to standing orders that were entered on an admission order set. After provider engagement, we proceeded to remove all admission order sets containing two-unit transfusions. Impact/lessons learned/results After order set removal, our one-unit transfusion rate rose to $86 \%$ and was sustained for 17 months. We learnt two primary lessons. First that CPOE and poor order set design combined to perpetuate poor ordering practices. Second that revisiting our hypothesis and engaging in thoughtful root cause analysis that included direct observation ultimately led to an effective, sustainable solution.

Discussion/spread Our study underscores the importance of executing root cause analysis on a microsystem level. We would expect the factors driving poor performance to be completely different on a service such as general internal medicine. Our study also highlights the potential pitfalls of CPOE and the importance of regular order set review to ensure adherence to current evidence.

\section{BACKGROUND}

Transfusion guidelines strongly support restrictive red blood cell (RBC) transfusion thresholds for almost all patient groups with the exception of acute coronary syndrome.
Implicit in restrictive transfusion strategies is the use of the smallest effective dose of RBC or single unit RBC transfusions. ${ }^{12}$ In 2014, Choosing Wisely Canada synthesised the evidence supporting one-unit rather than two-unit red cell transfusions and initiated a campaign to reduce the proportion of two-unit transfusions. Despite these clear guidelines, the proportion of one-unit transfusions on the oncology ward at London Health Sciences Centre (LHSC) remained low at $\sim 50 \%$. Other centres' efforts to improve rates of one-unit transfusion have shown that policy changes, audit \& feedback and clinical decision support can succeed when used in various combinations. Additionally, unpublished provincial data from the Ontario Regional Blood Coordinating Network have suggested that a laboratory technologist screening programme can effectively improve one-unit transfusion rates. It is, however, unknown to what degree these interventions impact provider/user workflow and contribute to phenomena such as alarm fatigue, change fatigue and feedback fatigue. In our study, with careful process observation we were able to identify an effective system design solution that avoided these drawbacks.

Several studies have attempted to increase the percentage of single unit transfusion orders. In one study, ${ }^{3}$ the group attempted to use policy change alone. This intervention was successful in modestly increasing the rate of single unit transfusion orders from $\sim 12 \%$ to $\sim 28 \%$; however, the sustainability of this improvement was unclear. Another study ${ }^{4}$ combined policy with standardisation: the blood bank would only dispense one unit of blood at a time except in pre-specified circumstances. This study was more successful-single unit transfusion orders increased from $25 \%$ to $84 \%$. Education via electronic clinical decision support was attempted in one study ${ }^{5}$ with modest success: the ratio of single to multiple unit transfusion orders improved from 0.34 to 1.2. Two 
studies have looked at audit and feedback, one combined with education alone ${ }^{6}$ and one with education plus policy change. ${ }^{7}$ A modest improvement was observed in the first study, as single unit transfusion orders rose from a baseline of $30 \%-50 \%$ to a postintervention rate of $70 \%-80 \%$; the second study demonstrated an improvement from $65 \%$ pre-intervention to $90 \%$ postintervention.

Examined in isolation, the above examples demonstrate that several interventions can potentially improve single unit RBC transfusion rates. Within a complex healthcare environment, however, many often unrelated projects are introduced concurrently, which risks inducing change fatigue. ${ }^{8}$ Depending on the nature of the interventions, phenomena such as alarm/popup fatigue, or feedback fatigue can further reduce effectiveness. ${ }^{9}$

The oncology unit at Victoria Hospital, LHSC is an academic tertiary care unit with 60 inpatient beds shared among medical oncology, haematology oncology and radiation oncology. The bulk of day-to-day physician care is provided by General Practitioner (GP) oncologists and nurse practitioners, with supervision by the most responsible physician. House staff and subspecialty fellows intermittently provide front line care. At the time of our project, our hospital was a little over a year into its implementation of computerised provider order entry (CPOE), and familiarity with its use was just starting to stabilise. The development of standardised order sets, called 'Power Plans', was part of the initial attraction of CPOE. Unintended, but somewhat predictable local consequences of $\mathrm{CPOE}$ implementation included provider workflow disruptions, and change fatigue. In part to mitigate and facilitate acceptance of CPOE, individual providers were allowed to modify standardised order sets and save these change as 'favourites' which could be shared with any number of other users. CPOE saw the exponential growth of favourites from standardised power plans.

Examination of our baseline transfusion data indicated that our baseline one-unit transfusion rate was $\sim 50 \%$ for the several months leading up to the project. Our aim was to increase the one-unit transfusion rate from $50 \%$ to $80 \%$ within 6 months.

\section{MATERIALS AND METHODS}

Our project utilised established quality improvement methodology based on the Model for Improvement. ${ }^{10}$ We began with a root cause analysis targeted at discovering contributing factors to two-unit transfusion orders. This analysis included: (1) a retrospective audit of the previous month's two-unit transfusions, (2) structured brainstorming by the study authors and (3) focused interviews with house staff and attending physicians. Using information obtained from these three sources, we constructed a fishbone/Ishikawa diagram. This diagram served as a comprehensive, structured list of all the possible factors potentially contributing to two-unit transfusions. The preliminary diagram was vetted through blood bank personnel to arrive at the final diagram (figure 1).

After completing our initial root cause analysis, we targeted selected factors in several phases. We first attempted an educational campaign with an educational email, and a Grand Rounds presentation focussing on improving awareness of the Choosing Wisely guidelines. The email message was sent to all oncology attending physicians once. The presentation was delivered once to an audience that included mainly house staff, nurse practitioners and GP oncologists, but did not specifically target the oncology attending physicians.

When these failed to demonstrate improvement, we began the second phase by executing a real-time audit. During this 1 week period, whenever a two-unit transfusion was ordered on the oncology ward, the receiving blood bank technologist phoned one of our authors $(\mathrm{AB})$, who then contacted the ordering physician to elicit the rationale for two units. This audit uncovered that virtually all of the two-unit transfusions were resulting from 'standing

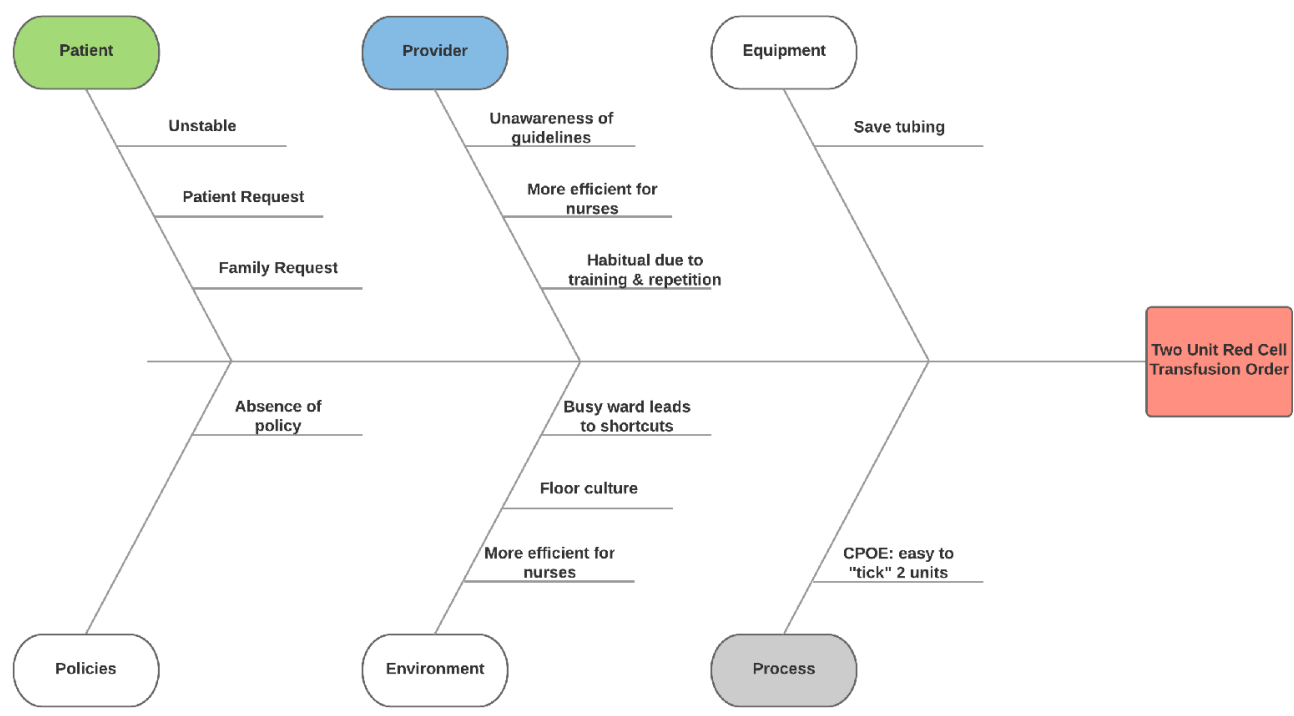

Figure 1 Ishikawa diagram of factors contributing to two-unit red cell transfusions. 
Percent One Unit Orders for Red Cell Transfusion

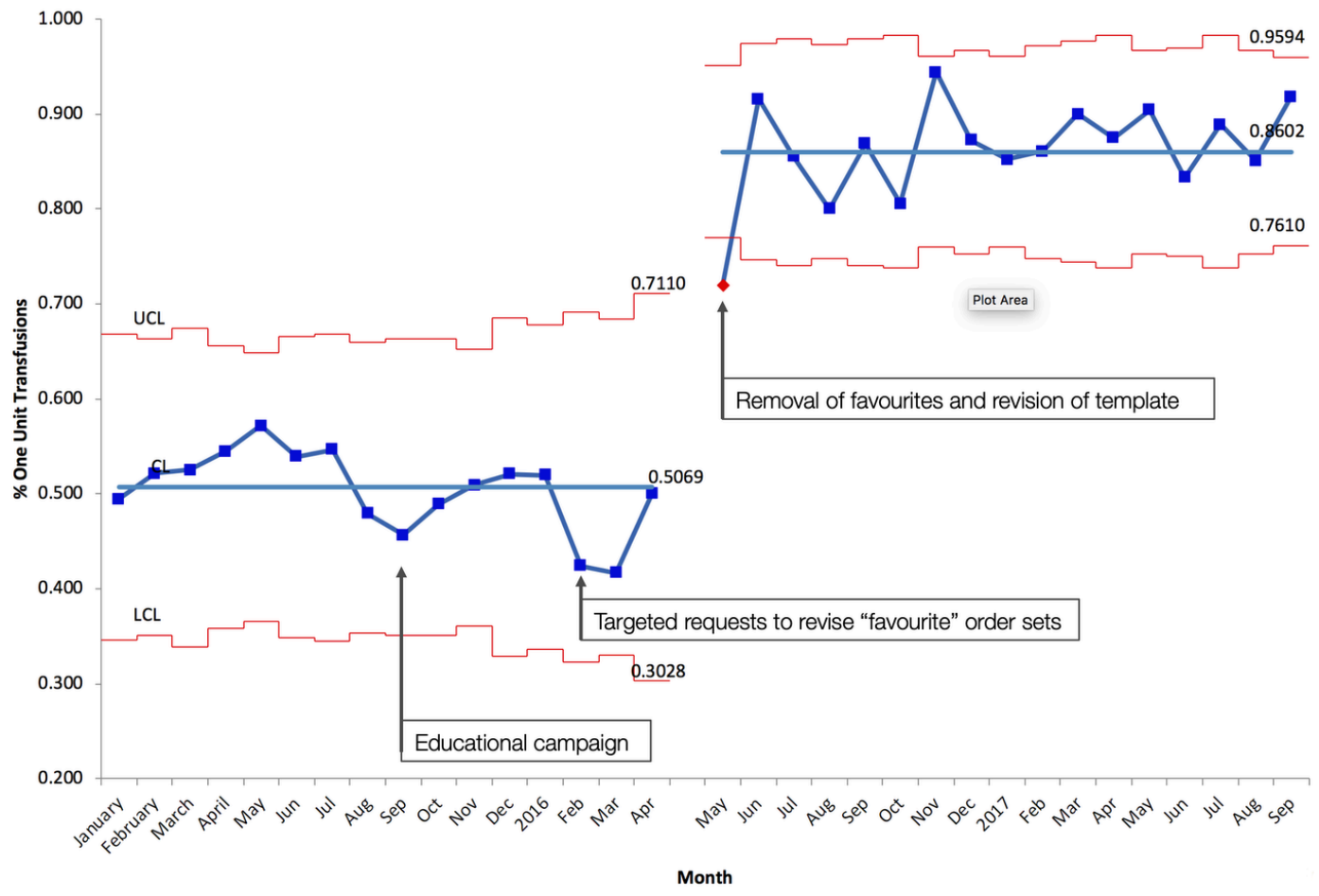

Figure 2 Statistical process control chart tracking percentage of single unit red cell transfusions over time.

orders' built into a master admission order set template called Haematology Oncology Admission that had been entered at the time of admission. The results of this audit prompted our second intervention: a targeted email to oncologists requesting the revision of personalised favourite order templates.

This intervention also failed to demonstrate improvement, thus our third phase involved focused oncologist interviews. We directly asked the oncologists why two-unit transfusions were included on their favourites; they acknowledged that they were aware of the guidelines, but had neglected to adjust their favourites once they were created. We brought the identified problem and guidelines to the attention of clinicians in a face-to-face group meeting; with their approval we proposed to remove all care set favourites containing two-unit transfusion orders on the oncology service. Our third and final intervention was the removal of favourite templates containing two-unit orders, and a revision of the master admission template to default to one unit.

Data on the outcome measure were collected continuously and automatically via blood bank electronic records. The data were aggregated and summarised monthly.

\section{RESULTS}

Data are displayed visually on a statistical process control chart (figure 2). The baseline from January to December 2015 show common cause variation only (no points outside of control limits), indicating a stable but poorly performing system operating outside of accepted guidelines for RBC transfusions. The mean proportion of one-unit transfusions during the baseline period was 50.7\%. Interventions \#1 and \#2 (email blitz and rounds presentation) resulted in no change to the system. Intervention \#3 (CPOE removal of favourites containing two-unit transfusion order, and revision of admission template to contain a one-unit transfusion order) resulted in an immediate and sustained change to the system. Postintervention, the mean proportion of one-unit transfusions rose to $86.0 \%$ and was sustained for the 17 months of ongoing data collection.

\section{DISCUSSION}

We learnt several key lessons during our study. The first was that a solution as simple as order set design can have a large impact, without the undesirable consequences of alarm fatigue/popup fatigue/change fatigue. Two recent studies demonstrated how the mere presence of an order on an admission order set makes it inherently more likely to be ordered. ${ }^{11} 12$ Interventions explored in the literature had either a modest (policy change alone) or unsustained (education alone) effect, or required a significant amount of effort to upkeep (audit and feedback). Through careful examination of our local context, we were able to identify a system-level change that will require minimal upkeep. Additionally, this relatively noninvasive intervention does not disrupt work flow; we would therefore expect minimal if any contribution to change/alert fatigue. What allowed us to implement such a change with almost no resistance was the care that we took in engaging the key stakeholders-the ordering oncologists. Though our initiative was supported by 


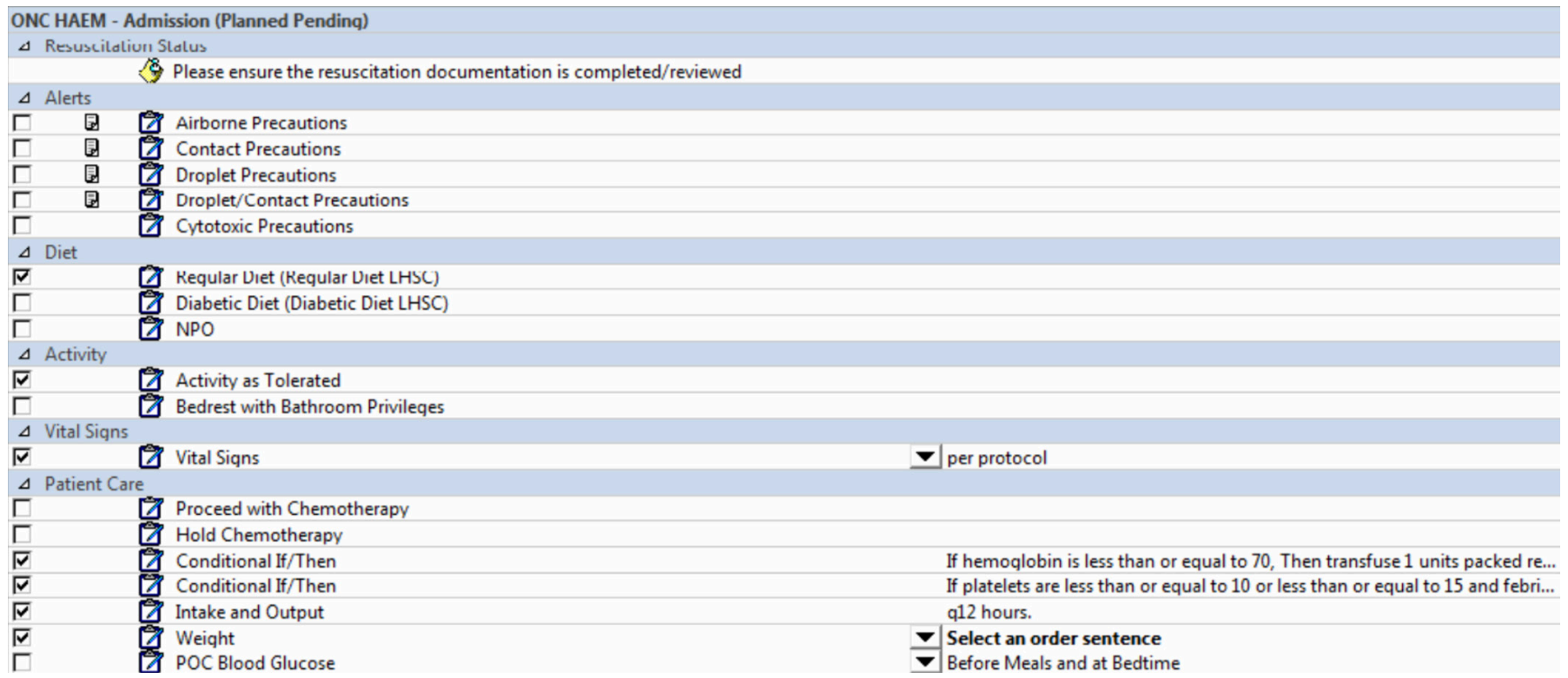

Figure 3 Standardised haematology/oncology admission template post revision. The template may be modified and saved as a personalised 'favourite'.

guidelines, our clinicians may have felt that their patient population was an exception. Engaging them early and allowing them to voice concerns allowed us to proceed confidently when no objections arose.

The second lesson was that CPOE is a double-edged sword. CPOE was touted to be a solution to many problems such as transcription errors and non evidence-based ordering practices. At our centre, CPOE allows for a standardised template to be modified and saved as a personal favourite, which would then be quickly available for subsequent use by that individual as well as the sharing with other providers (figure 3). Of note, when we interviewed providers, they acknowledged they were aware that guidelines recommended one-unit transfusions, but had never gone back to revisit previously established order sets or often did not have the technical knowledge to change their favourites. Like user passwords, most electronic choices are never revisited unless required by the system. Furthermore, while our CPOE allows the modification of power plans after discussion with the developer, favourite folders are not monitored and the sheer number (estimated at $>15000$ in our system) makes them challenging to change. The dramatic improvement that occurred when we took it on ourselves to remove offending order sets supports the assertion that it was neither awareness nor adherence to archaic doctrine that was driving clinician behaviour in this case.

The third lesson was the importance of continually re-evaluating our hypotheses to arrive at a true root cause. Our preliminary analysis of literature and our retrospective audit led us to the wrong conclusion-that unawareness of modern transfusion guidelines and/or adherence to the obsolete two-unit RBC transfusion doctrine were primarily responsible for the high proportion of two-unit transfusions. It was only after directly observing the process that we were able to uncover the true root cause-facilitation and perpetuation of two-unit transfusions by our CPOE system and poorly designed order set. Directly observing the process, or 'going to the gemba' to use Lean terminology, is a well-studied and well-accepted technique for uncovering root causes. At the extreme, some advanced Lean practitioners have argued that if the solution to a problem is not obvious, then you have not done enough observation. In our study, this revelation led to the ultimately successful intervention. Without the continuous revisitation, the first two lessons and the project success would not have been possible.

Our study had several limitations. Our study was not only single centre, but a specific clinical microsystem within a single centre. Gaining acceptance from small group of clinicians for the intervention was straightforward once the problem in the order set was identified. Another unit that is adjacent to the pilot unit may have a completely set of contextual circumstances that are driving clinician behaviour and therefore require different interventions. We are keeping this in mind, in fact, as we spread our campaign to the general internal medicine units, where we would expect that very few transfusion orders would arise from standing orders, and house staff do the majority of the ordering. This is not so much a limitation, but is rather the nature of quality improvement, which is extremely context-dependent. Another limitation was that we did not collect explicit process metrics to support our hypotheses. We could have evaluated our educational intervention to demonstrate uptake and understanding; however, this would have required a prohibitive amount of effort. Furthermore, the focused interviews we conducted supported that clinicians were aware of the guidelines, but that factors other than education were at play.

In conclusion, we were able to discover a low upkeep, effective intervention to improve the proportion of one-unit red cell transfusions on the oncology ward at our 
centre; we recommend that similar projects in other units and other centres should similarly carry out a detailed root cause analysis that includes direct observation. This technique can inspire a context-specific intervention that is minimally invasive compared with its alternatives. Lastly, as more hospitals employ CPOE, it is important to recognise its pitfalls and establish a regular review and update process to ensure that order sets meet current guidelines.

Contributors AG and IC-Y were involved in all aspects of study design, analysis and manuscript preparation. $A B$ was involved in survey of root cause analysis, educational interventions and initial draft of manuscript. LA was involved in data analysis in all phases of the project and review draft manuscript.

Funding The authors have not declared a specific grant for this research from any funding agency in the public, commercial or not-for-profit sectors.

Competing interests None declared.

Patient consent for publication Not required.

Provenance and peer review Not commissioned; externally peer reviewed.

Open access This is an open access article distributed in accordance with the Creative Commons Attribution Non Commercial (CC BY-NC 4.0) license, which permits others to distribute, remix, adapt, build upon this work non-commercially, and license their derivative works on different terms, provided the original work is properly cited, appropriate credit is given, any changes made indicated, and the use is non-commercial. See: http://creativecommons.org/licenses/by-nc/4.0/.

\section{REFERENCES}

1. Carson JL, Grossman BJ, Kleinman S, et al. Red blood cell transfusion: a clinical practice guideline from the AABB*. Ann Intern Med 2012;157:49-58.
2. Retter A, Wyncoll D, Pearse R, et al. Guidelines on the management of anaemia and red cell transfusion in adult critically ill patients. $\mathrm{Br} \mathrm{J}$ Haematol 2013;160:445-64.

3. Leahy MF, Roberts H, Mukhtar SA, et al. A pragmatic approach to embedding patient blood management in a tertiary hospital. Transfusion 2014;54:1133-45.

4. Berger MD, Gerber B, Arn K, et al. Significant reduction of red blood cell transfusion requirements by changing from a double-unit to a single-unit transfusion policy in patients receiving intensive chemotherapy or stem cell transplantation. Haematologica 2012:97:116-22.

5. McKinney ZJ, Peters JM, Gorlin JB, et al. Improving red blood cell orders, utilization, and management with point-of-care clinical decision support. Transfusion 2015;55:2086-94.

6. Yang WW, Thakkar RN, Gehrie EA, et al. Single-unit transfusions and hemoglobin trigger: relative impact on red cell utilization. Transfusion 2017;57:1163-70.

7. Baer VL, Henry E, Lambert DK, et al. Implementing a program to improve compliance with neonatal intensive care unit transfusion guidelines was accompanied by a reduction in transfusion rate: a pre-post analysis within a multihospital health care system. Transfusion 2011;51:264-9.

8. McMillan K, Perron A. Nurses amidst change: the concept of change fatigue offers an alternative perspective on organizational change. Policy Polit Nurs Pract 2013;14:26-32.

9. Wright A, Aaron S, Seger DL, et al. Reduced effectiveness of interruptive drug-drug interaction alerts after conversion to a commercial electronic health record. J Gen Intern Med 2018;33:1868-76.

10. Berwick DM. Developing and testing changes in delivery of care. Ann Intern Med 1998;128:651-6.

11. Leis B, Frost A, Bryce R, et al. Standard admission order sets promote ordering of unnecessary investigations: a quasirandomised evaluation in a simulated setting. BMJ Qual Saf 2017;26:938-40.

12. Leis JA, Shojania KG. A primer on PDSA: executing plan-dostudy-act cycles in practice, not just in name. BMJ Qual Saf 2017;26:572-7. 